\title{
Prognostic significance of nucleophosmin mutations and FLT3 internal tandem duplication in adult patients with cytogenetically normal acute myeloid leukemia
}

\author{
Yeo-Kyeoung Kim ${ }^{1}$, Hee-Nam Kim ${ }^{2}$, Se Ryeon Lee ${ }^{3}$, Jae-Sook Ahn ${ }^{1}$, Deok-Hwan Yang ${ }^{1}$, Je-Jung Lee ${ }^{1}$, \\ Il-Kwon Lee ${ }^{2}$, Myung-Geun Shin ${ }^{4}$, Hyeoung-Joon Kim ${ }^{1,2}$ \\ ${ }^{1}$ Department of Hematology/Oncology, Research Institute of Medical Sciences, Chonnam National University Medical School, \\ Gwangju, ${ }^{2}$ Genome Research Center for Hematopoietic Diseases, Chonnam National University Hwasun Hospital, Hwasun, \\ ${ }^{3}$ Department of Hematology/Oncology, Korea University Anam Hospital, Seoul, ${ }^{4}$ Department of Laboratory Medicine, Chonnam \\ National University Medical School, Gwangju, Korea
}

p-ISSN 1738-7949 / e-ISSN 2092-9129 DOI: $10.5045 /$ kjh.2010.45.1.36 Korean J Hematol 2010;45:36-45.

Received on February 18, 2010

Revised on March 5, 2010

Accepted on March 9, 2010

This work was supported by a research grant from the Korea Health 21 R\&D Project, Ministry of Health and Welfare, Republic of Korea (A010385) and the Research Institute of Medical Sciences, Chonnam National University (2007-CURIMS-DR007).

\section{Correspondence to}

Hyeoung-Joon Kim, M.D., Ph.D.

Hematology/Oncology Clinics, Chonnam National University Hwasun Hospital, 160 Ilsim-ri, Hwasun-eup, Hwasun-gun 519-809, Korea

Tel: + 82-61-379-7637

Fax: +82-61-379-7628

E-mail: hjoonk@chonnam.ac.kr

(C) 2010 The Korean Journal of Hematology

\section{Background}

Nucleophosmin (NPM1) gene and fms-like tyrosine kinase 3 gene-internal tandem duplication (FLT3-ITD) mutations are the most frequent mutations in patients with cytogenetically normal (CN)-AML. We analyzed the prognostic impact of these mutations and their interactions in adults with CN-AML.

Methods

NPM1 mutation (NPM1mut) and FLT3-ITD mutation (FLT3-ITD+) were analyzed by GeneScan and PCR assays of bone marrow samples obtained from 121 adult patients with $\mathrm{CN}$-AML (age $\leq 60$ years at diagnosis).

Results

The incidence of FLT3-ITD + was higher in the NPM1 mut group than in the wild-type NPM1 gene (NPM1 wt) group. The patients were divided according to their mutation status into the NPM1 mut/FLT3-ITD (isolated NPM1 mut), NPM1 mut/FLT3-ITD+ or NPM1 wt/FLT3-ITD-, and NPM1wt/FLT3-ITD+ (isolated FLT3-ITD+) groups. The isolated NPM1 mut group showed significantly better clinical outcomes in terms of relapse rate, 5-year relapse-free survival (RFS), and overall survival (OS) than the other groups. In contrast, the isolated FLT3-ITD+ group had a higher relapse rate and shorter RFS and OS than the other groups. The 5-year RFS rate was much higher among the patients who underwent allogeneic stem cell transplantation (alloSCT) than among those treated with high-dose cytarabine chemotherapy (HDAC) only as consolidation therapy in the isolated NPM1 mut group and the NPM1 mut/FLT3-ITD+ or NPM1wt/FLT3-ITD- group.

Conclusion

Adult patients with CN-AML carrying isolated NPM1 mut and isolated FLT3-ITD+ exhibit different clinical outcomes than those with NPM1 mut/FLT3-ITD+ or NPM1 wt/FLT3-ITD-. Although isolated NPM1 mut leads to favorable clinical outcomes of CN-AML, the role of alloSCT in such patients remains to be considered.

Key Words NPM1, FLT3-ITD, Acute myeloid leukemia, Normal karyotype

\section{INTRODUCTION}

AML is a clinically and genetically heterogeneous disease. Three cytogenetically defined risk groups (favorable, inter- mediate, and adverse) are used to design risk-adapted treatment protocols for patients with AML. However, about $35-50 \%$ of the successfully karyotyped patients lack clonal chromosomal aberrations [1], and the prognostic implications have not been clearly established for cytogenetically normal (CN)-AML [2]. Therefore, it is important to develop a molec-

\footnotetext{
This is an Open Access article distributed under the terms of the Creative Commons Attribution Non-Commercial License (http://creativecommons.org/licenses/by-nc/3.0)
} which permits unrestricted non-commercial use, distribution, and reproduction in any medium, provided the original work is properly cited. 
ular-level genetic approach for discriminating between prognostically different subsets of CN-AML.

In the recent years, CN-AML has been identified as a heterogeneous disease with mutations of the nucleophosmin (NPMI) and fms-like tyrosine kinase 3 (FLT3) genes. NPM1, encoded by the $N P M 1$ gene on chromosome $5 \mathrm{q} 35$, is a multifunctional nucleocytoplasmic shuttling protein localized primarily in the nucleolus but shuttles rapidly between the nucleus and the cytoplasm. The protein appears to be important for various cellular processes. NPM may assist in ribosomal protein assembly [3] and maintain genomic stability through its participation in DNA repair [4]. It also plays a crucial role in cell cycle regulation and apoptosis via its interactions with tumor suppressor p53 and alternate reading frame protein $[5,6]$.

The NPM1 gene frequently acts as a target of chromosomal translocations and causes the cytoplasmic dislocation of proteins in various types of leukemia and lymphoma, indicating its role in malignant transformation. Recent studies have demonstrated that aberrant cytoplasmic localization of NPM (NPMc+) in leukemic blasts is associated with mutations at exon-12 of the NPM1 gene [7-9]. NPM1 exon-12 mutations can encode mutant proteins with a novel nuclear export signal (NES) motif inserted at the $C$-terminus and disruption of the nucleolar localization signal due to mutations of tryptophan residues 288 and 290 [7, 8]. Such mutations are classified according to the type of NES motif inserted into the mutant protein. In adult NPMc+ AML, mutation 'A' (tandem duplication of TCTG) accounts for approximately $80 \%$ of all the NPMc+ cases [9]. Mutations at NPM1 exon-12 and the resultant shift of $N P M 1$ into the cytoplasm are found in approximately $35 \%$ of the adults with AML. One of the most frequent mutations seen in $\mathrm{CN}-\mathrm{AML}$ is $N P M 1$ mutation (NPM1mut), found in $45.7-64 \%$ of the patients. Besides the characteristic biological features such as increased frequency of monocytic leukemia and distinctive gene expression profiles, patients with CN-AML carrying isolated NPM1mut show better clinical outcomes in terms of the responsiveness to chemotherapy or disease-free survival (DFS) than those with the wild-type $N P M 1$ (NPM1wt) gene [9-12].

FLT3 belongs to the class III receptor tyrosine kinase family. It is expressed in early hematopoietic progenitors and its dimerization by the FLT3 ligand induces growth control signals in normal hematopoiesis. The FLT3 gene maps to chromosome band 13q12 [13], and an internal tandem duplication (ITD) of the gene (FLT3-ITD) has been detected in $20-30 \%$ of the young adults with AML [14]. The duplication involves a segment of the juxtamembrane domain-coding sequence, which frequently involves exon-14 and rarely intron-14 or exon-15, and is always in-frame [14]. In support of the potential role of this mutation in leukemogenesis, FLT3-ITD from patients with AML has been shown to induce autonomous proliferation in cytokine-dependent cell lines. In contrast to NPM1mut, patients with isolated FLT3-ITD mutation (FLT3-ITD+) show poor clinical outcomes, similar to those with poor-risk cytogenetics $[9,12,14]$.

Several studies have attempted to identify the interactions between these frequent mutations and their prognostic implications for patients with CN-AML. On the basis of these reports, the recently updated National Comprehensive Cancer Network (NCCN) Guidelines [15] recommend that isolated NPMImut and FLT3-ITD+ CN-AML should be regarded as better-risk and poor-risk cytogenetics, respectively; hence, transplantation as a postremission therapy should be reserved until relapse in patients with CN-AML carrying isolated NPM1mut. In contrast, patients with FLT3-ITD+ CN-AML should be enrolled in clinical trials as the standard therapy on account of their poor prognosis. Nonetheless, controversy exists as to the prognostic significance of these two mutations in patients with CN-AML $[9,16,17]$. Therefore, we examined the incidence and interactions of NPMlmut and FLT3-ITD+ in such patients and assessed the validity of the newly proposed risk-adapted treatment strategies based on these mutations.

\section{MATERIALS AND METHODS}

\section{Patients and treatment protocol}

Diagnostic bone marrow (BM) samples from 121 adult patients with CN-AML (age $\leq 60$ years) who had received at least one cycle of intensive induction chemotherapy were analyzed retrospectively for the presence of NPM1mut and FLT3-ITD+. To establish CN-AML, 20 or more metaphase cells from the samples had to test positive for normal karyotypes [18]. Approval was obtained from the institutional review board for this procedure. All patients gave written informed consent for both the treatment and the cryopreservation of BM.

All enrolled patients received intensive remission induction therapy consisting of 3 days of idarubicin (IDA) at $12 \mathrm{mg} / \mathrm{m}^{2} /$ day and 7 days of cytarabine at $100 \mathrm{mg} / \mathrm{m}^{2} /$ day or $N^{4}$-behenoyl-1-D-arabinofuranosylcytosine (BH-AC; 300 $\mathrm{mg} / \mathrm{m}^{2} /$ day for patients younger than 40 years, $200 \mathrm{mg} / \mathrm{m}^{2} /$ day for patients older than 40 years). If the patients failed to achieve complete remission (CR) after the first round of induction chemotherapy, they received reinduction chemotherapy using the same regimen. Patients achieving CR received three courses of high-dose cytarabine chemotherapy (HDAC; $3 \mathrm{~g} / \mathrm{m}^{2}$ every $12 \mathrm{~h} /$ day on days 1,3 , and 5 ) for consolidation. These patients were allowed to proceed to allogeneic (alloSCT) or autologous stem cell transplantation (autoSCT) after one or two courses of HDAC consolidation therapy.

\section{Mutational analysis}

Genomic DNA was extracted from the diagnostic BM samples, such as cryopreserved mononuclear cells, by using a DNA blood minikit (QIAamp; Qiagen, Valencia, CA, USA) according to the manufacturer's protocol.

For NPM1mut analysis, NPM1 exon-12 was amplified by genomic PCR using primers 5'-TCTGAGTATAAATTTTCT TGGAGTCA-3' (sense) and 5'-ACCAAGCAAAGGGTGGAG TT-3' (antisense). The reaction mixture contained $1.25 \mathrm{pmol}$ 
of each primer, $50 \mathrm{ng}$ of genomic DNA, $250 \mu \mathrm{M}$ dNTPs, and $0.5 \mathrm{U}$ f-taq polymerase (Solgent, Daejeon, Korea) in the buffer provided by the manufacturer. Amplification was performed in a thermal cycler (PTC 200; MJ Research, Inc., Waltham, MA, USA), and the PCR fragments were purified (GENEALL PCR Purification Kit; General Biosystem, Seoul, Korea). The sequencing reactions were analyzed by using a sequencer (ABI 3100) and cycle sequencing kit (BigDye Terminator; Applied Biosystems, Foster City, CA, USA).

For FLT3-ITD+ analysis, wild-type FLT3 exon-11 and exon-12 were amplified by genomic PCR using primers 5'-CAATTTAGGTATGAAAGCC-3' (sense) and 5'-CTTTCA GCATTTTGACGGCAACC-3' (antisense). The reaction mixture contained $2.5 \mathrm{mM}$ dNTPs, $2.5 \mathrm{mM} \mathrm{MgCl}_{2}, 0.5 \mu \mathrm{M}$ of each primer, and $0.5 \mathrm{U}$ f-taq polymerase in a total volume of $20 \mu \mathrm{L}$. The samples were amplified by initial denaturation at $95^{\circ} \mathrm{C}$ for $5 \mathrm{~min}$, followed by 35 cycles of $94^{\circ} \mathrm{C}$ for 30 sec, $53^{\circ} \mathrm{C}$ for $1 \mathrm{~min}$, and $72^{\circ} \mathrm{C}$ for $2 \mathrm{~min}$, and final extension at $72^{\circ} \mathrm{C}$ for $10 \mathrm{~min}$. The PCR products $(10 \mu \mathrm{L})$ were resolved on $6 \%$ polyacrylamide gels, stained with ethidium bromide, and photographed under ultraviolet light.

\section{Statistical analysis}

The response to initial therapy was evaluated after induction or after salvage chemotherapy. The definition of CR followed the recommended criteria [19]. Relapse was defined as the reappearance of blasts post-CR in the peripheral blood or BM. Relapse-free survival (RFS) endpoints, measured from the date of documented CR, included relapse, patient death from any cause, and alive in $\mathrm{CR}$ at last follow-up (censored). The overall survival (OS) endpoints, measured from the date of diagnosis, were death from any cause and alive at last follow-up (censored) [19]. RFS before transplantation and OS before transplantation were also assessed to eliminate confounding bias and were defined as the time without relapse, death, or transplantation from the date of $\mathrm{CR}$ and the time from diagnosis to death or transplantation, respectively.

For between-group comparisons, Fisher's exact test (categorical data) and the Mann-hitney U test (continuous data) were used. Categorical data were compared among three groups defined by the NPM1 and FLT3-ITD mutation status using one-way analysis of variance (ANOVA) and the post hoc Tukey's honestly significant difference (HSD) test. Continuous variables were compared among the three groups by using the Kruskal-Wallis test. RFS and OS were analyzed by means of Kaplan-Meier survival curve estimates and logrank tests to compare differences in the distribution of survival for the three groups. Multivariate analysis using forward conditional selection of variables was performed with the Cox's proportional-hazards model to analyze the influence of high WBC count $\left(>50 \times 10^{9} / \mu \mathrm{L}\right.$ versus $\left.\leq 50 \times 10^{9} / \mu \mathrm{L}\right)$, secondary AML (versus de novo AML), alloSCT, autoSCT, and the NPM1 and FLT3-ITD mutation status or the interaction of these mutations. A $P$-value of less than 0.05 was considered statistically significant. All statistical computations were performed by using SPSS software version 17.0 (SPSS, Inc.,
Chicago, IL, USA).

\section{RESULTS}

\section{Patient characteristics}

The median age of the 57 male and 64 female patients was 44 years (range, 15-60 years). All patients received intensive remission induction chemotherapy. Fifty-three (43.8\%) and five $(4.1 \%)$ of the patients underwent alloSCT and autoSCT, respectively. The median follow-up time was 11.8 months (range, 0.6-86.1 months).

Among the patients, 65 (53.7\%) had NPMlmut at exon-12. All patients carrying NPM1mut had the type A mutation. Details of the presenting features by the NPM1mut status are listed in Table 1. The median peripheral WBC count at diagnosis was significantly higher in the $N P M 1$ mut group than in the $N P M$ w wt group $(P=0.03)$. Furthermore, the $N P M$ lmut group showed a higher incidence of FLT3-ITD+ than the unmutated group $(P=0.04)$.

To follow the current NCCN guidelines, we categorized the patients into three groups: NPMImut/FLT3-ITD-, NPM1mut/

Table 1. Patient characteristics according to the NPM1 mut status.

\begin{tabular}{|c|c|c|c|c|}
\hline Parameter & NPM1wt & NPM1mut & Total AML & $P$ \\
\hline No. of patients & $56(46.3)$ & 65 (53.7) & 121 & \\
\hline Age $(y r)$ & $43(15-60)$ & $47(16-60)$ & $44(15-60)$ & 0.65 \\
\hline Sex & & & & 0.86 \\
\hline Male & $27(48.2)$ & $30(46.2)$ & $57(47.1)$ & \\
\hline Female & 29 (51.8) & 35 (53.8) & $64(52.9)$ & \\
\hline FAB subtype & & & & NA \\
\hline Mo & $3(5.4)$ & $2(3.1)$ & $5(4.1)$ & \\
\hline M1 & $12(21.4)$ & $12(18.5)$ & $24(19.8)$ & \\
\hline M2 & $18(32.1)$ & $20(30.8)$ & $38(31.4)$ & \\
\hline M4 & $9(16.1)$ & 9 (13.8) & $18(14.9)$ & \\
\hline M5 & $4(7.1)$ & $10(15.4)$ & $14(11.6)$ & \\
\hline M6 & $2(3.6)$ & $4(6.2)$ & $6(5.0)$ & \\
\hline M7 & $3(5.4)$ & 0 & $3(2.5)$ & \\
\hline $\begin{array}{r}\text { Secondary } \\
\text { leukemia }\end{array}$ & $4(7.1)$ & $8(12.3)$ & $12(9.9)$ & \\
\hline Unknown & $1(1.8)$ & 0 & $1(0.8)$ & \\
\hline WBC $\left(\times 10^{9} / \mathrm{L}\right)$ & $\begin{array}{c}11.8 \\
(0.5-333.2)\end{array}$ & $\begin{array}{c}29.7 \\
(1.3-190.4)\end{array}$ & $\begin{array}{c}25.0 \\
(0.5-333.2)\end{array}$ & 0.03 \\
\hline $\mathrm{Hb}(\mathrm{g} / \mathrm{dL})$ & $\begin{array}{c}7.2 \\
(3.1-10.6)\end{array}$ & $\begin{array}{c}8.4 \\
(4.9-12.9)\end{array}$ & $\begin{array}{c}7.6 \\
(3.1-12.9)\end{array}$ & 0.23 \\
\hline Platelets $\left(\times 10^{9} / \mathrm{L}\right)$ & $46(19-87)$ & $62(5-150)$ & $61(5-150)$ & 0.22 \\
\hline BM blasts (\%) & 78 (20-99) & $80(20-100)$ & $80(20-100)$ & 0.50 \\
\hline AlloSCT & $24(42.9)$ & $29(44.6)$ & $53(43.8)$ & 0.86 \\
\hline AutoSCT & $4(7.1)$ & $1(1.5)$ & $5(4.1)$ & 0.18 \\
\hline FLT3-ITD+ & $16(28.6)$ & $30(46.2)$ & $46(38.0)$ & 0.04 \\
\hline
\end{tabular}

The values represent either the number (percentage) or the median (range).

Abbreviations: NPM1wt, wild-type nucleophosmin gene; NPM1 mut, nucleophosmin gene mutation NA, not applicable; $\mathrm{BM}$, bone marrow; alloSCT, allogeneic stem cell transplantation autoSCT; autologous stem cell transplantation FLT3-ITD, fms-like tyrosine kinase 3 gene-internal tandem duplication. 
FLT3-ITD+ or NPM1wt/FLT3-ITD-, NPM1wt/FLT3-ITD+. Table 2 shows the baseline characteristics of these groups. There was no difference among the groups with regard to age and gender. The $N P M 1 \mathrm{wt} / F L T 3-\mathrm{ITD}+$ group showed the highest initial WBC count; however, there was no statistical significance among the groups.

\section{Prognostic impact of NPM1}

Of the 121 patients, 93 (76.9\%) achieved CR. Among these, 19 patients failed to achieve CR after the first round of induction chemotherapy and therefore received reinduction chemotherapy (Table 3). There was no difference in the remission rate to induction chemotherapy between the patients with and those without NPM1mut $(76.9 \%$ versus $76.8 \%, P=0.99)$. Among the patients who achieved CR, the NPM1mut group showed a lower relapse rate before SCT (18.0\% versus $34.9 \%, P=0.06)$ and lower overall relapse rate (36.0\% versus $47.6 \%, P=0.26$ ), although the differences were not statistically significant. Before undergoing SCT, the

Table 2. Patient characteristics according to the NPM1mut and FLT3-ITD+ status.

\begin{tabular}{|c|c|c|c|c|}
\hline Parameter & $\begin{array}{l}\text { NPM1mut/ } \\
\text { FLT3-ITD- }\end{array}$ & $\begin{array}{l}\text { NPM1wt/ } \\
\text { FLT3-ITD- } \\
\text { NPM1mut/ } \\
\text { FLT3-ITD+ }\end{array}$ & $\begin{array}{l}\text { NPM1wt/ } \\
\text { FLT3-ITD+ }\end{array}$ & $P$ \\
\hline No. of patients & 35 (28.9) & 70 (57.9) & $16(13.2)$ & \\
\hline Age (yr) & $50(23-60)$ & $43(16-60)$ & $44(15-54)$ & 0.49 \\
\hline Sex & & & & 0.51 \\
\hline Male & $19(54.3)$ & $32(45.7)$ & $6(37.5)$ & \\
\hline Female & $16(45.7)$ & $38(54.3)$ & $10(62.5)$ & \\
\hline FAB subtypes & & & & NA \\
\hline Mo & $2(5.7)$ & $3(4.3)$ & 0 & \\
\hline M1 & $5(14.3)$ & $15(21.4)$ & $4(25.0)$ & \\
\hline M2 & $15(42.9)$ & $20(28.6)$ & $3(18.8)$ & \\
\hline M4 & $3(8.6)$ & 9 (12.9) & $6(37.5)$ & \\
\hline M5 & $5(14.3)$ & $8(11.4)$ & $1(6.3)$ & \\
\hline M6 & $2(5.7)$ & $4(5.7)$ & 0 & \\
\hline M7 & 0 & $2(2.9)$ & $1(6.3)$ & \\
\hline $\begin{array}{r}\text { Secondary } \\
\text { leukemia }\end{array}$ & $3(8.6)$ & $8(11.4)$ & $1(6.3)$ & \\
\hline Unknown & 0 & $1(1.4)$ & 0 & \\
\hline WBC $\left(10^{9} / \mathrm{L}\right)$ & $\begin{array}{c}29.7 \\
(1.3-178.8)\end{array}$ & $\begin{array}{c}20.7 \\
(0.5-333.2)\end{array}$ & $\begin{array}{c}37.9 \\
(6.4-74.9)\end{array}$ & 0.38 \\
\hline $\mathrm{Hb}(\mathrm{g} / \mathrm{dL})$ & $\begin{array}{c}9.2 \\
(4.9-12.9)\end{array}$ & $\begin{array}{c}7.5 \\
(3.1-10.8)\end{array}$ & $\begin{array}{c}8.7 \\
(7.1-9.2)\end{array}$ & 0.42 \\
\hline Platelets $\left(10^{9} / \mathrm{L}\right)$ & $66.6(5-150)$ & $45(19-116)$ & $68(25-87)$ & 0.50 \\
\hline BM blasts (\%) & 74 (20-90) & $80(20-100)$ & $79(20-98)$ & 0.27 \\
\hline AlloSCT & $13(37.1)$ & $34(48.6)$ & $6(37.5)$ & 0.57 \\
\hline AutoSCT & $1(2.9)$ & $3(4.3)$ & $1(6.3)$ & 0.82 \\
\hline
\end{tabular}

The values represent either the number (percentage) or the median (range).

Abbreviations: NPM1wt, wild-type nucleophosmin gene; NPM1 mut, nucleophosmin gene mutation FLT3-ITD, fms-like tyrosine kinase 3-internal tandem duplication; NA, not applicable; BM, bone marrow; alloSCT, allogeneic stem cell transplantation; autoSCT; autologous stem cell transplantation.
NPM1mut group showed a significantly higher rate of 4-year RFS (45.4\% versus $0 \%, P=0.00)$ and OS (59.8\% versus $15.6 \%$, $P=0.04$ ) than the $N P M 1$ wt group (Fig. 1A). Overall RFS and OS also showed a trend toward better prognosis in the NPM1mut group (Fig. 1B).

In the subgroup analysis, the overall RFS was significantly longer among the patients who underwent alloSCT than among the nontransplant patients $(P=0.01)$ in the $N P M 1 \mathrm{wt}$ group (Fig. 2A). On the other hand, in the NPMImut group, a trend for longer RFS was observed among the alloSCT patients than the nontransplant patients, but the difference was not statistically significant $(P=0.34$; Fig. 2B). Although few patients underwent autoSCT $(\mathrm{N}=5)$, this procedure did not influence the overall relapse rate, RFS, and OS (data not shown).

According to the multivariate analysis for the overall RFS, NPM1mut (versus NPM1wt, OR=0.49, 95\% CI=0.26-0.92, $P=0.03$ ), FLT3-ITD- (versus FLT3-ITD+, OR=0.32, 95\% CI= $0.17-0.60, P=0.00$ ), and alloSCT (versus chemotherapy only, $\mathrm{OR}=0.41,95 \% \mathrm{CI}=0.22-0.77, P=0.01)$ were independently associated with longer overall RFS, after adjusting for high WBC counts $\left(\geq 50 \times 10^{9} / \mu \mathrm{L}\right.$ versus $\left.<50 \times 10^{9} / \mu \mathrm{L}\right)$ and secondary AML (versus de novo AML) (Table 4). In the multivariate analysis for the overall OS, NPMImut failed to be an independent prognostic factor, whereas FLT3-ITD- and alloSCT were independent, favorable prognostic factors (Table 4).

\section{Prognostic impact of the interactions between NPM1 mut and FLT3-ITD+}

In agreement with the previous reports, our study showed that the FLT3-ITD+ status was significantly associated with $N P M 1$ mut in terms of the incidence and clinical outcomes;

Table 3. Treatment outcomes according to the NPM1 mut status.

\begin{tabular}{lcccc}
\multicolumn{1}{c}{ Parameter } & NPM1wt & NPM1 mut & Total AML & $P$ \\
\hline No. of patients & $56(46.3)$ & $65(53.7)$ & 121 & \\
CR1 $^{\text {a) }}$ & $34(60.7)$ & $40(61.5)$ & $74(61.2)$ & 0.94 \\
CR total $^{\text {b) }}$ & $43(76.8)$ & $50(76.9)$ & $93(76.9)$ & 0.99 \\
Resistant & $12(21.4)$ & $14(21.5)$ & $26(21.5)$ & 0.99 \\
Early death & $1(1.8)$ & $1(1.5)$ & $2(1.7)$ & NA \\
Relapse before SCT & $15(34.9)$ & $9(18.0)$ & $24(25.8)$ & 0.06 \\
Overall relapse & $20(47.6)^{c)}$ & $18(36.0)$ & $\left.38(41.3)^{c}\right)$ & 0.26 \\
4-yr RFS before SCT & 0 & $45.4 \pm 1.4$ & $24.7 \pm 10.3$ & 0.00 \\
4-yr OS before SCT & $15.6 \pm 12.5$ & $59.8 \pm 11.3$ & $41.8 \pm 9.0$ & 0.04 \\
5-yr RFS & $20.2 \pm 15.1$ & $49.1 \pm 8.7$ & $39.5 \pm 7.8$ & 0.18 \\
5-yr OS & $44.2 \pm 9.1$ & $52.5 \pm 7.7$ & $48.8 \pm 5.8$ & 0.29 \\
\hline
\end{tabular}

The values represent either the number (percentage) or the mean \pm SD.

a) Patients achieved CR after one course of remission induction chemotherapy, ${ }^{\text {b) }}$ Patients achieved CR regardless of induction chemotherapy, ${ }^{c}$ One patient was missing due to follow-up loss. Abbreviations: NPM1wt, wild-type nucleophosmin gene; NPM1 mut, nucleophosmin gene mutation $\mathrm{CR}$, complete remission; NA, not applicable; SCT, stem cell transplantation; RFS, relapse-free survival; OS, overall survival. 


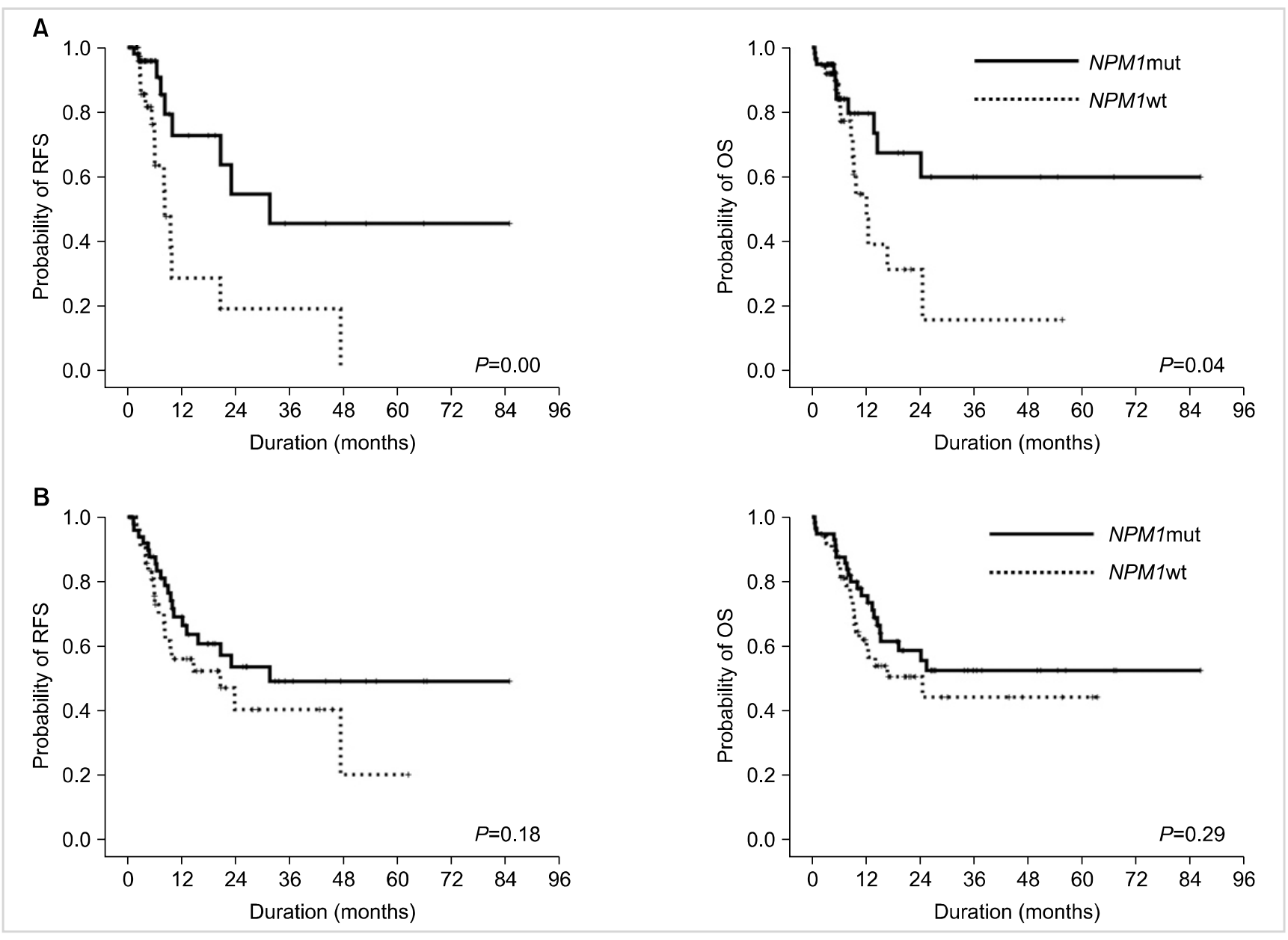

Fig. 1. Kaplan-Meier analysis of CN-AML according to the NPM1 mut status. (A) RFS and OS before SCT. (B) Overall RFS and OS. Abbreviations: CN, cytogenetically normal; NPM1 mut, nucleophosmin gene mutation; NPM1wt, wild-type nucleophosmin gene; RFS, relapse-free survival; OS, overall survival; SCT, stem cell transplantation.

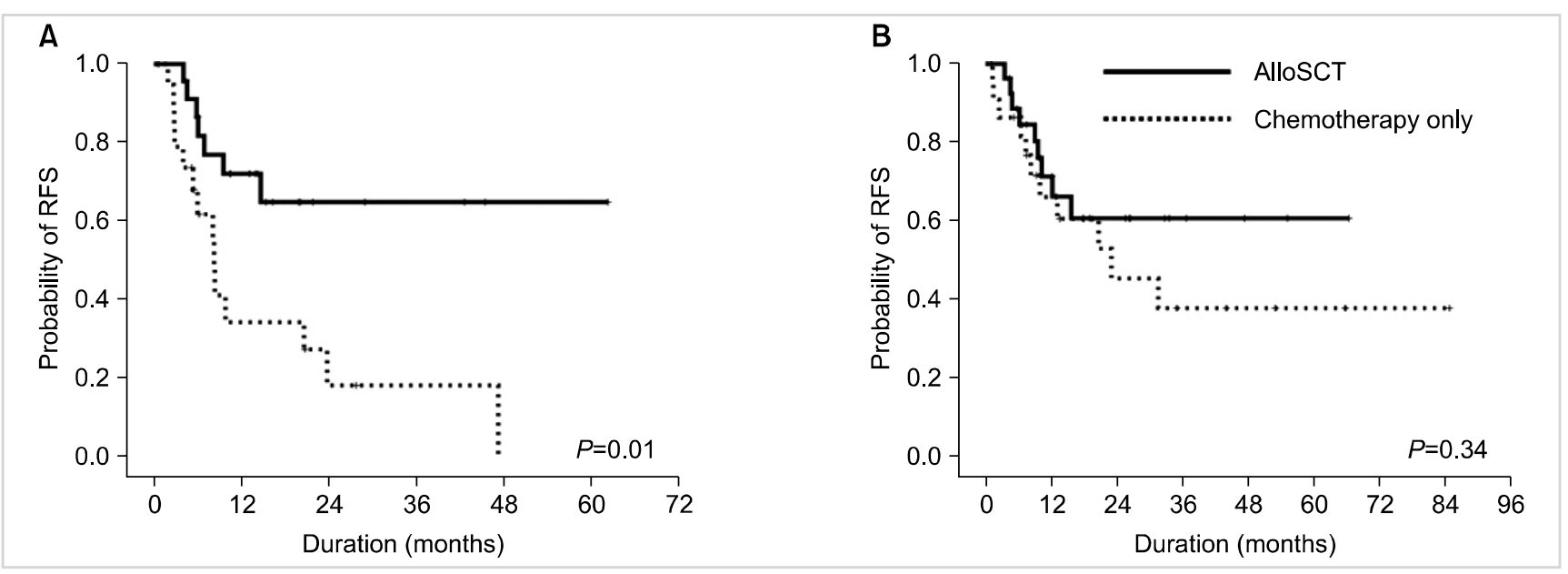

Fig. 2. RFS analysis of the alloSCT patients versus the chemotherapy-alone patients according to the NPM1 mut status. (A) Overall RFS in the NPM1 wt group. The overall RFS was significantly longer among the alloSCT patients than among the chemotherapy-alone patients $(P=0.01)$. (B) Overall RFS in the NPM1 mut group. A trend of longer survival was observed among the alloSCT patients than among the chemotherapy-alone patients, but the difference was not statistically significant $(P=0.34)$. Abbreviations: NPM1 mut, nucleophosmin gene mutation; NPM1 wt, wild-type nucleophosmin gene; RFS, relapse-free survival; alloSCT, allogeneic stem cell transplantation.

hence, we assessed the prognostic impact of the three groups according to the NPMImut and FLT3-ITD+ status (Table 5). The highest remission rate was found in the NPM1mut/
FLT3-ITD- group (80.0\%); however, the differences among the groups were not statistically significant. The relapse rate before SCT was higher in the NPM1wt/FLT3-ITD+ group 
Table 4. Multivariable analysis for outcome according to the NPM1mut and FLT3-ITD+ status.

\begin{tabular}{|c|c|c|c|c|}
\hline \multirow[b]{2}{*}{ Variable } & \multicolumn{2}{|l|}{ RFS } & \multicolumn{2}{|l|}{ OS } \\
\hline & $\begin{array}{c}\text { OR } \\
(95 \% \mathrm{Cl})\end{array}$ & $P$ & $\begin{array}{c}\text { OR } \\
(95 \% \mathrm{Cl})\end{array}$ & $P$ \\
\hline \multicolumn{5}{|l|}{ NPM1wt } \\
\hline NPM1mut & $\begin{array}{c}0.49 \\
(0.26-0.92)\end{array}$ & 0.03 & NA & $\mathrm{NA}$ \\
\hline \multicolumn{5}{|l|}{ FLT3-ITD+ } \\
\hline FLT3-ITD - & $\begin{array}{c}0.32 \\
(0.17-0.60)\end{array}$ & 0.00 & $\begin{array}{c}0.42 \\
(0.23-0.77)\end{array}$ & 0.01 \\
\hline \multicolumn{5}{|l|}{$\begin{array}{l}\text { Interaction of NPM1 } \\
\text { and FLT3-ITD }\end{array}$} \\
\hline \multicolumn{5}{|l|}{ NPM1mut/FLT3-ITD - } \\
\hline NPM1wt/FLT3-ITD - & $\begin{array}{c}0.30 \\
(0.14-0.64)\end{array}$ & 0.00 & $\begin{array}{c}0.32 \\
(0.15-0.69)\end{array}$ & 0.00 \\
\hline \multicolumn{5}{|l|}{ NPM1mut/FLT3-ITD - } \\
\hline NPM1wt/FLT3-ITD+ & $\begin{array}{c}0.16 \\
(0.06-0.40)\end{array}$ & 0.00 & $\begin{array}{c}0.20 \\
(0.08-0.50)\end{array}$ & 0.00 \\
\hline \multicolumn{5}{|l|}{ AlloSCT } \\
\hline \multicolumn{5}{|l|}{ No } \\
\hline Yes & $\begin{array}{c}0.43 \\
(0.23-0.81)\end{array}$ & 0.01 & $\begin{array}{c}0.35 \\
(0.18-0.66)\end{array}$ & 0.00 \\
\hline
\end{tabular}

Abbreviations: NPM1wt, wild-type nucleophosmin gene; NPM1 mut, nucleophosmin gene mutation FLT3-ITD, fms-like tyrosine kinase 3 gene-internal tandem duplication; RFS, relapse-free survival; OS, overall survival; OR, odds ratio; $95 \% \mathrm{Cl}, 95 \%$ confidence interval; NA, not applicable; alloSCT, allogeneic stem cell transplantation.

(41.7\%) than in the NPM1mut/FLT3-ITD- group (21.4\%) and the other groups (NPM1wt/FLT3-ITD- or NPM1mut/ FLT3-ITD+, 24.5\%). Furthermore, the overall relapse rate was significantly higher in the isolated FLT3-ITD+ group $(83.3 \%)$ than in the isolated NPM1mut $(28.6 \%)$ and the other groups (38.5\%). The post hoc test also revealed that the NPM1wt/FLT3-ITD+ group had a significantly higher overall relapse rate than the NPM1mut/FLT3-ITD- $(P=0.00)$ or the other groups $(P=0.01)$. The highest 5-year RFS and OS were observed in the isolated $N P M 1$ mut group regardless of SCT, followed by the NPM1wt/FLT3-ITD- or NPM1mut/FLT3-ITD+ group and the isolated FLT3-ITD+ groups (Fig. 3).

We also conducted further analysis for the overall RFS following alloSCT compared with chemotherapy alone as a consolidation therapy. In the isolated NPM1mut group, 13 patients (37.1\%) underwent alloSCT whereas $20(57.1 \%)$ received chemotherapy alone. The CR rate in the alloSCT group and chemotherapy-alone group was $69.2 \%$ and $55 \%$, respectively $(P=0.49)$. Twelve patients $(92.3 \%)$ achieved $\mathrm{CR}$ at transplantation. One $(7.7 \%)$ was refractory to all three courses of previous remission induction chemotherapy; in spite of alloSCT, the patient could not achieve CR and died of pneumonia 3 months after SCT. Peripheral blood stem cells collected from sibling donors (s-PBSCs, $\mathrm{N}=11$ ), unrelated bone marrow stem cells ( $\mathrm{u}-\mathrm{BMs}, \mathrm{N}=1$ ), and unrelated PBSCs ( $\mathrm{u}$-PBSCs, $\mathrm{N}=1$ ) were used as the hematopoietic stem cell
Table 5. Treatment outcomes according to the NPM1mut and FLT3-ITD+ status.

\begin{tabular}{|c|c|c|c|c|}
\hline Parameter & $\begin{array}{l}\text { NPM1mut/ } \\
\text { FLT3-ITD- }\end{array}$ & $\begin{array}{l}\text { NPM1wt/ } \\
\text { FLT3-ITD- } \\
\text { NPM1 mut/ } \\
\text { FLT3-ITD+ }\end{array}$ & $\begin{array}{l}\text { NPM1wt/ } \\
\text { FLT3-ITD+ }\end{array}$ & $P$ \\
\hline No. of patients & 35 (28.9) & $70(57.9)$ & $16(13.2)$ & \\
\hline $\mathrm{CR} 1^{\mathrm{a})}$ & $21(60.0)$ & $43(61.4)$ & $10(62.5)$ & 1.00 \\
\hline CR total ${ }^{b)}$ & $28(80.0)$ & $53(75.7)$ & $12(75.0)$ & 0.78 \\
\hline Resistant & $6(17.1)$ & $16(22.9)$ & $4(25.0)$ & 0.78 \\
\hline Early death & $1(2.9)$ & $1(1.4)$ & 0 & 0.75 \\
\hline $\begin{array}{l}\text { Relapse } \\
\text { before SCT }\end{array}$ & $6(21.4)$ & $13(24.5)$ & $5(41.7)$ & 0.40 \\
\hline Overall relapse & 8 (28.6) & $20(38.5)^{\mathrm{c})}$ & $10(83.3)$ & 0.00 \\
\hline $\begin{array}{l}\text { 5-yr RFS } \\
\text { before SCT }\end{array}$ & $52.4 \pm 15.7$ & $11.9 \pm 10.7$ & NA & 0.00 \\
\hline $\begin{array}{l}5-y r \text { OS } \\
\text { before SCT }\end{array}$ & $61.4 \pm 12.0$ & $34.8 \pm 14.8$ & 0 & 0.02 \\
\hline 5-yr RFS & $60.5 \pm 10.8$ & $35.4 \pm 12.3$ & 0 & 0.00 \\
\hline $5-y r$ OS & $62.6 \pm 9.1$ & $47.1 \pm 8.7$ & NA & 0.00 \\
\hline
\end{tabular}

The values represent either the number (percentage) or the mean \pm SD.

a) Patients achieved CR after one course of remission induction chemotherapy, ${ }^{\text {b) }}$ Patients achieved CR regardless of induction chemotherapy, ${ }^{\text {c) }}$ One patient was missing due to follow-up loss. Abbreviations: NPM1wt, wild-type nucleophosmin gene; NPM1 mut, nucleophosmin gene mutation FLT3-ITD, fms-like tyrosine kinase 3 gene-internal tandem duplication; CR, complete remission; SCT, stem cell transplantation; RFS, relapse-free survival; NA, not applicable; OS, overall survival.

sources. Conditioning regimens consisted of $\mathrm{Flu} / \mathrm{Bu}$ (fludarabine at $30 \mathrm{mg} / \mathrm{m}^{2} /$ day for 6 days and busulfan at $3.2 \mathrm{mg} /$ $\mathrm{kg} /$ day for 4 days, N=9), TBI/Cy (total body irradiation at $13.2 \mathrm{~Gy}$ in 4 days with cyclophosphamide at $60 \mathrm{mg} /$ day in 2 days, $\mathrm{N}=3$ ), and $\mathrm{Bu} / \mathrm{Cy}$ (busufan with cyclophosphamide, $\mathrm{N}=1$ ). The median number of infused CD34+ cells was $5.3 \times$ $10^{6} / \mathrm{kg}$ (range=0.7-13.2). As depicted in Fig. 4, alloSCT significantly influenced the longer (5-year) RFS in the isolated NPMImut group (alloSCT versus chemotherapy alone, $87.5 \%$. versus $42.7 \%, P=0.03$; Fig. $4 \mathrm{~A}$ ). In addition, we conducted a subgroup analysis of 21 patients who achieved CR after one cycle of induction chemotherapy ( 9 in alloSCT group, 11 in chemotherapy-alone group, and 1 in autoSCT group). The alloSCT group showed a significantly lower rate of overall relapse $(0 \%$ versus $36.4 \%, P=0.04)$ and higher 5 -year overall RFS (100\% versus $52.5 \%, P=0.04)$ than the chemotherapy-alone group. Although statistically insignificant, the patients with $N P M 1 \mathrm{wt} / F L T 3$ - ITD- or NPM1mut/FLT3ITD+ who underwent alloSCT (5-year RFS=63.6\%) showed better overall RFS than the chemotherapy-alone patients (15.1\%, $P=0.12$; Fig. 4B). Among the patients with isolated FLT3-ITD+, the overall RFS in the alloSCT group $(\mathrm{N}=6)$ was not significantly different from that in the chemotherapy-alone group ( $\mathrm{N}=9$; Fig. 4C). s-PBSCs ( $=4$ ), u-PBSCs $(\mathrm{N}=1)$, and $\mathrm{u}-\mathrm{BMs}(\mathrm{N}=1)$ were used as the stem cell sources and all patients underwent alloSCT at CR status. The con- 

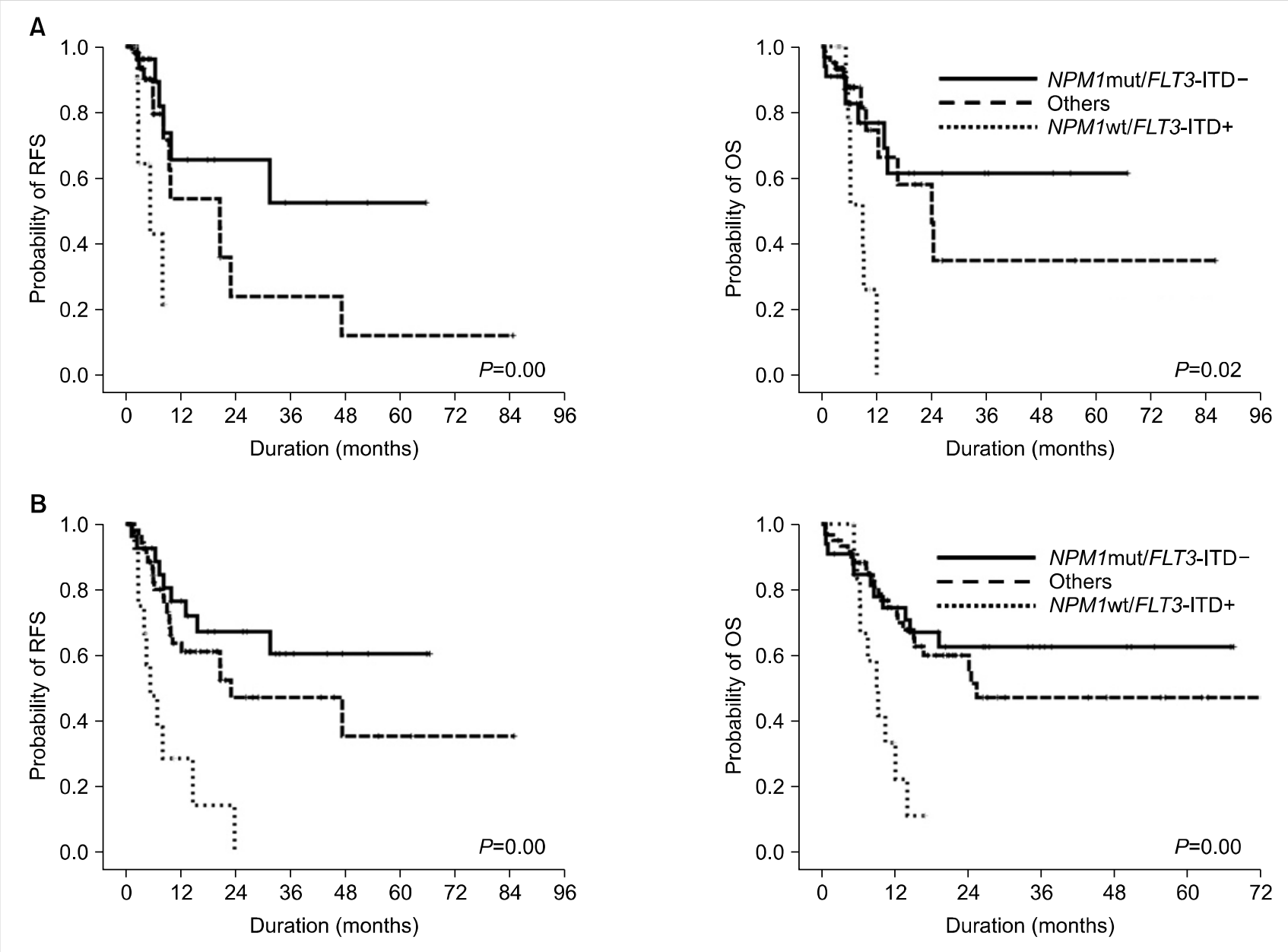

Fig. 3. Kaplan-Meier analysis of CN-AML for RFS and OS according to the combined NPM1mut and FLT3-ITD+ status. (A) RFS and OS before transplantation. (B) Overall RFS and OS. Abbreviations: CN, cytogenetically normal; NPM1 mut, nucleophosmin gene mutation; NPM1wt, wild-type nucleophosmin gene; FLT3-ITD, fms-like tyrosine kinase 3 gene-internal tandem duplication; RFS, relapse-free survival; OS, overall survival.

ditioning regimens consisted of $\mathrm{Flu} / \mathrm{Bu}(\mathrm{N}=4)$ and $\mathrm{TBI} / \mathrm{Cy}$ $(\mathrm{N}=2)$. The median number of infused $\mathrm{CD} 34+$ cells was $4.5 \times$ $10^{6} / \mathrm{kg}$ (range $=2.6-7.8$ ). Four patients in the alloSCT group died of leukemic relapse, and there was no significant correlation between the relapse rate or RFS and the conditioning regimen or the stem cell source.

The multivariate analysis for the overall RFS and OS also identified the combination of NPM1 and FLT3-ITD mutations and alloSCT as independent prognostic factors after adjusting for high WBC count $\left(\geq 50 \times 10 \% / \mu \mathrm{L}\right.$ versus $\left.<50 \times 10^{9} / \mu \mathrm{L}\right)$ and secondary AML (versus de novo AML) (Table 4).

\section{DISCUSSION}

We evaluated the prevalence and prognostic impact of NPMImut and FLT3-ITD+ and their interactions in adult patients with CN-AML. The incidence of $N P M 1$ mut and FLT3-ITD+ was $53.7 \%$ and $38 \%$, respectively, suggesting that they are frequent molecular events in such patients. The incidence of FLT3-ITD+ was higher in the NPMImut group than in the $N P M 1$ wt group. Previous studies have reported a considerable correlation between these two mutations, suggesting that they are secondary events from a primary process that predisposes myeloid stem and progenitor cell errors in DNA replication $[10,11]$. Others have demonstrated that the $N P M 1$ mut/allele ratio is higher than the FLT3-ITD+/wild-type ratio, suggesting that NPM1mut occurs prior to FLT3-ITD+ in cases with both mutations [9].

According to previous studies, patients with CN-AML carrying $N P M 1$ mut show a higher rate of CR, suggesting that these patients are more sensitive to chemotherapeutic agents $[11,12,20]$. These studies assumed that NPMc+ may interact with and sequester nuclear factor kappaB (NF- $\kappa \mathrm{B}$ ), contributing to the maintenance and survival of malignant clones and an impaired response to chemotherapy in the cytoplasm, leading to its inactivation and reduced DNA binding [20]. However, in other studies, patients younger than 60 years and pediatric patients did not show a significantly different CR rate between $N P M 1$ wt and $N P M 1$ mut $[9,16,17]$. In the present study, there were no significant differences in the CR rate between NPM1mut and NPM1wt. Although the patients with NPM1mut/FLT3-ITD- CN-AML showed a slightly higher $\mathrm{CR}$ rate than the other groups, the differ- 

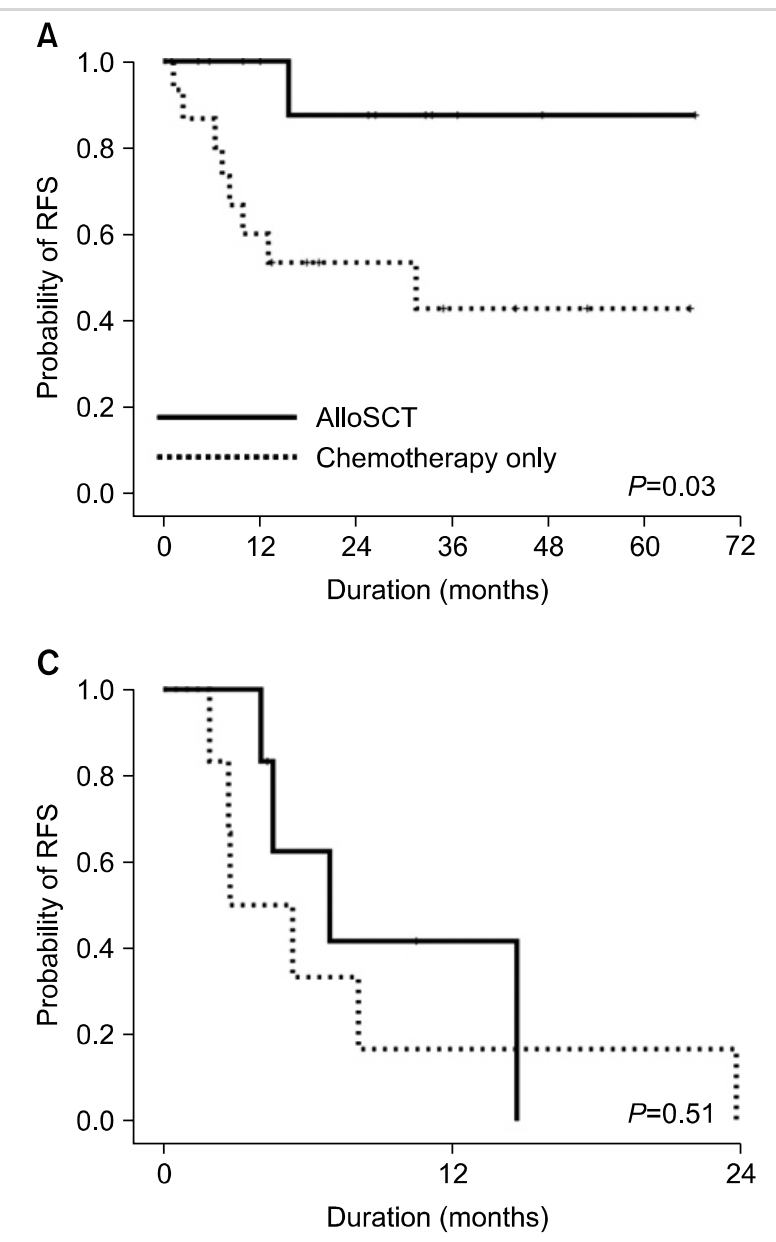

ences were not statistically significant.

On the other hand, we demonstrated that the NPM1mut group had better RFS and OS before SCT than the NPMlwt group, although the differences were not statistically significant. A recent study has demonstrated that non-A type NPM1mut individuals show different clinical outcomes compared with those having the type A mutation [21]. In the present study, however, all the NPMlmut individuals possessed type A mutations. In the multivariate analysis to evaluate other factors influencing the overall RFS and OS, FLT3-ITD+ and alloSCT treatment were identified as independent prognostic factors (besides the NPMlmut status).

For CN-AML, the proper risk-stratified postremission therapy has not yet been determined. Previous studies on young adult patients with CN-AML have reported a 4-year DFS of $48.5 \%$ with alloSCT [22] and 5-year DFS of $28-41 \%$ with repeated courses of HDAC consolidation [23, 24]. Hence, transplant-based options generally afford a lesser risk of relapse and higher DFS as consolidation for the patients. However, this benefit is accompanied by a treatment-related mortality of $15-25 \%$ [25]. To balance the treatment-related toxicity and risk of relapse, it is crucial to define distinct clinical and molecular subtypes that influence the prognosis of CN-AML.

It was previously reported that event-free survival (EFS)

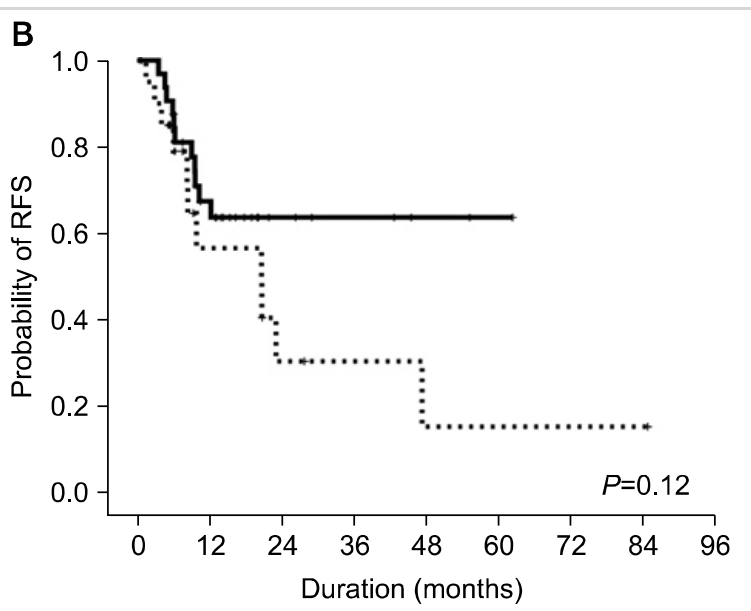

Fig. 4. RFS analysis of the alloSCT patients versus the chemotherapyalone patients according to the combined NPM1 mut and FLT3-ITD+ status. (A) NPM1 mut/FLT3-ITD- (isolated NPM1 mut) group. (B) NPM1 mut/ FLT3-ITD+ or NPM1 wt/FLT3-ITD- group. (C) NPM1wt/FLT3-ITD+ (isolated FLT3-ITD+) group. In the NPM1 mut/FLT3-ITD- group, the alloSCT patients had significantly longer RFS than the chemotherapy- alone patients (A). The NPM1 mut/FLT3-ITD+ or NPM1wt/FLT3-ITD- group also displayed a longer trend of RFS with alloSCT (B). In contrast, the patients with NPM1wt/FLT3-ITD+ showed significantly shorter RFS and alloSCT did not improve the outcome of this subset (C). Abbreviations: NPM1 mut, nucleophosmin gene mutation; NPM1wt, wild-type nucleophosmin gene; FLT3-ITD, fms-like tyrosine kinase 3 gene-internal tandem duplication; RFS, relapse-free survival; alloSCT, allogeneic stem cell transplantation. and DFS are better in patients with NPMc+/FLT3-ITD- than in those with other CN-AML subtypes [9, 12]. Some studies have also shown that the 5-year EFS and OS of patients with NPMc+/FLT3-ITD- are comparable to those with favorable cytogenetics $[16,17]$, suggesting that this group of patients could be considered a favorable group. Others have reported that the RFS and OS are significantly better in the NPMc+/FLT3-ITD- group than the other groups, and suggested that alloSCT has no effect on the RFS and OS in this subset of CN-AML [11]. Therefore, the NCCN guidelines for AML consider patients with isolated NPM1mut as a favorable-risk group and recommended multiple cycles of HDAC as a reasonable option. Further, patients carrying isolated FLT3-ITD+ constitute a poor-risk cytogenetic group that should be considered for clinical trials or early alloSCT [15]. However, other studies have reported variable results about the clinical outcomes according to the presence of these mutations; the FLT3-ITD+ status was prognostically significant only in the patients with $N P M 1$ wt $[18,19]$.

In our three-group analysis, the isolated $N P M 1$ mut group had a significantly better overall relapse rate and the highest rate of 5-year RFS and OS, regardless of SCT. Further, in this group, alloSCT significantly increased the 5-year RFS rate. For patients carrying favorable cytogenetics such as $\mathrm{t}(8 ; 21)$ or inv (16), multiple courses of HDAC are known 
to lead to $50-60 \%$ of 4 -year DFS [24, 26]. In the present study, the 5 -year RFS was $42.7 \%$ for the patients treated only with HDAC consolidation in the isolated NPM1mut group, lower than that among patients with favorable cytogenetics. The patients who underwent alloSCT, however, showed a significantly better 5-year RFS rate (87.5\%) than the chemotherapy-alone patients in the isolated $N P M 1 \mathrm{mut}$ group. Therefore, the role of alloSCT in patients with NPMImut/ FLT3-ITD- CN-AML should be reconsidered.

In contrast, the isolated FLT3-ITD+ group showed poor clinical outcomes in terms of the overall relapse rate, RFS, and OS, regardless of SCT. All patients relapsed or died within 2 years after diagnosis, and alloSCT did not change their RFS.

In the $N P M 1 \mathrm{wt} / F L T 3-I T D-$ or $N P M 1 \mathrm{mut} / F L T 3-I T D+$ group, the 5-year RFS increased remarkably (but not significantly) with alloSCT compared with the chemotherapyalone patients, who showed a remarkably lower rate of 5-year RFS (15.1\%) than the patients who underwent alloSCT (63.6\%). Therefore, alloSCT seems to be a reasonable option for this subset of patients.

In summary, adult patients ( $\leq 60$ years) with $\mathrm{CN}$-AML carrying isolated NPM1mut and FLT3-ITD+ show different clinical outcomes than those bearing both mutated or wild-type NPM1 and FLT3-ITD. Furthermore, isolated NPM1mut is associated with favorable clinical outcomes in patients with CN-AML; however, the efficacy of alloSCT as a treatment option for this group of patients remains to be determined.

\section{REFERENCES}

1. Mrózek K, Heinonen K, Bloomfield CD. Clinical importance of cytogenetics in acute myeloid leukaemia. Best Pract Res Clin Haematol 2001;14:19-47.

2. Slovak ML, Kopecky KJ, Cassileth PA, et al. Karyotypic analysis predicts outcome of preremission and postremission therapy in adult acute myeloid leukemia: a Southwest Oncology Group/ Eastern Cooperative Oncology Group Study. Blood 2000;96: 4075-83.

3. Szebeni A, Olson MO. Nucleolar protein B23 has molecular chaperone activities. Protein Sci 1999;8:905-12.

4. Lee SY, Park JH, Kim S, Park EJ, Yun Y, Kwon J. A proteomics approach for the identification of nucleophosmin and heterogeneous nuclear ribonucleoprotein $\mathrm{C} 1 / \mathrm{C} 2$ as chromatin-binding proteins in response to DNA double-strand breaks. Biochem J 2005;388:7-15.

5. Colombo E, Marine JC, Danovi D, Falini B, Pelicci PG. Nucleophosmin regulates the stability and transcriptional activity of p53. Nat Cell Biol 2002;4:529-33.

6. Bertwistle D, Sugimoto M, Sherr CJ. Physical and functional interactions of the Arf tumor suppressor protein with nucleophosmin/B23. Mol Cell Biol 2004;24:985-96.

7. Falini B, Bolli N, Shan J, et al. Both carboxy-terminus NES motif and mutated tryptophan(s) are crucial for aberrant nuclear export of nucleophosmin leukemic mutants in NPMc+ AML. Blood
2006;107:4514-23.

8. Falini B, Nicoletti I, Martelli MF, Mecucci C. Acute myeloid leukemia carrying cytoplasmic/mutated nucleophosmin (NPMc+ AML): biologic and clinical features. Blood 2007;109:874-85.

9. Thiede C, Koch S, Creutzig E, et al. Prevalence and prognostic impact of NPM1 mutations in 1485 adult patients with acute myeloid leukemia (AML). Blood 2006;107:4011-20.

10. Falini B, Mecucci C, Tiacci E, et al. Cytoplasmic nucleophosmin in acute myelogenous leukemia with a normal karyotype. N Engl J Med 2005;352:254-66.

11. Döhner K, Schlenk RF, Habdank M, et al. Mutant nucleophosmin (NPM1) predicts favorable prognosis in younger adults with acute myeloid leukemia and normal cytogenetics: interaction with other gene mutations. Blood 2005;106:3740-6.

12. Gale RE, Green C, Allen C, et al. The impact of FLT3internal tandem duplication mutant level, number, size, and interaction with NPM1 mutations in a large cohort of young adult patients with acute myeloid leukemia. Blood 2008;111:2776-84.

13. Rosnet O, Schiff C, Pébusque MJ, et al. Human FLT3/FLK2 gene: cDNA cloning and expression in hematopoietic cells. Blood 1993;82:1110-9.

14. Kottaridis PD, Gale RE, Frew ME, et al. The presence of a FLT3 internal tandem duplication in patients with acute myeloid leukemia (AML) adds important prognostic information to cytogenetic risk group and response to the first cycle of chemotherapy: analysis of 854 patients from the United Kingdom Medical Research Council AML 10 and 12 trials. Blood 2001;98:1752-9.

15. National Comprehensive Cancer Network. Version 2. 2010. (Accessed January 8, 2010, at http://www.nccn.org/professionals/ physician_gls/PDF/aml.pdf).

16. Brown P, McIntyre E, Rau R, et al. The incidence and clinical significance of nucleophosmin mutations in childhood AML. Blood 2007;110:979-85.

17. Hollink IH, Zwaan CM, Zimmermann M, et al. Favorable prognostic impact of NPM1 gene mutations in childhood acute myeloid leukemia, with emphasis on cytogenetically normal AML. Leukemia 2009;23:262-70.

18. Mrózek K, Carroll AJ, Maharry K, et al. Central review of cytogenetics is necessary for cooperative group correlative and clinical studies of adult acute leukemia: the Cancer and Leukemia Group B experience. Int J Oncol 2008;33:239-44.

19. Cheson BD, Bennett JM, Kopecky KJ, et al. Revised recommendations of the international working group for diagnosis, standardization of response criteria, treatment outcomes, and reporting standards for therapeutic trials in acute myeloid leukemia. J Clin Oncol 2003;21:4642-9.

20. Cilloni D, Messa F, Rosso V, et al. Increase sensitivity to chemotherapeutical agents and cytoplasmatic interaction between NPM leukemic mutant and NF-kappaB in AML carrying NPM1 mutations. Leukemia 2008;22:1234-40.

21. Koh Y, Park J, Bae EK, et al. Non-A type nucleophosmin 1 gene mutation predicts poor clinical outcome in de novo adult acute myeloid leukemia: differential clinical importance of NPM1 mutation according to subtype. Int J Hematol 2009;90:1-5.

22. Suciu S, Mandelli F, de Witte T, et al. Allogeneic compared with autologous stem cell transplantation in the treatment of patients younger than 46 years with acute myeloid leukemia (AML) in first 
complete remission (CR1): an intention-to-treat analysis of the EORTC/GIMEMAAML-10 trial. Blood 2003;102:1232-40.

23. Farag SS, Ruppert AS, Mrózek K, et al. Outcome of induction and postremission therapy in younger adults with acute myeloid leukemia with normal karyotype: a cancer and leukemia group B study. J Clin Oncol 2005;23:482-93.

24. Bloomfield CD, Lawrence D, Byrd JC, et al. Frequency of prolonged remission duration after high-dose cytarabine intensification in acute myeloid leukemia varies by cytogenetic subtype.
Cancer Res 1998;58:4173-9.

25. Estey E, Döhner H. Acute myeloid leukaemia. Lancet 2006;368: 1894-907.

26. Byrd JC, Mrózek K, Dodge RK, et al. Pretreatment cytogenetic abnormalities are predictive of induction success, cumulative incidence of relapse, and overall survival in adult patients with de novo acute myeloid leukemia: results from Cancer and Leukemia Group B (CALGB 8461). Blood 2002;100:4325-36. 